\title{
Fabrication and characterization of hexadecyl acrylate cross-linked phase change microspheres
}

https://doi.org/10.1515/epoly-2020-0008

Received June 22, 2019; accepted December 11, 2019.

\begin{abstract}
Microspheres with phase change properties were fabricated by polymerization of hexadecyl acrylate (HA) and different cross-linking agents. The samples were characterized by scanning electron microscope (SEM), Fourier transform infrared spectroscopy (FTIR), thermal gravimetric analysis (TGA). The results show that, the samples that added cross-linking agents have a smooth surface and the latent heat of them is different. The experiments show that all of the cross-linked copolymer shells can be made into temperature controlled release microspheres. These materials can be potentially applied in the field of thermal energy storage. $\beta$-tricalcium phosphate was encapsulated in microspheres to obtain one with a fast release effect. It will effectively promote bone conduction when these microspheres were implanted into a bone defect. This microsphere can be used for orthopedic implant or coating of instrument in the future.
\end{abstract}

Keywords: microspheres; phase change materials; crosslinking agents; fast release; properties

\section{Introduction}

Energy storage is a hot research topic in recent years. In particular, phase-change thermal storage technology is an important technology that makes rational and effective use of existing energy, optimizes the use of renewable

\footnotetext{
* Corresponding author: Xingxiang Zhang, Tianjin Municipal Key Laboratory of Advanced Fiber and Energy Storage Technology, Tianjin 300387, China; School of Material Science and Engineering, Tianjin Polytechnic University, Tianjin 300387, China, e-mail: zhangpolyu@aliyun.com, tel.: 0086-22-83955054 Haoran Yun, Tianjin Municipal Key Laboratory of Advanced Fiber and Energy Storage Technology, Tianjin 300387, China; School of Material Science and Engineering, Tianjin Polytechnic University, Tianjin 300387, China
}

energy and enhances energy efficiency (1,2). Phase change thermal storage materials use the latent heat of phase change to achieve the energy storage and utilization $(3,4)$. The realization of phase change energy storage technology relies on phase change materials (PCMs). PCM is a kind of material which can change its phase when it absorbs or releases heat, and the latent heat reflects the heat absorption and heat release ability of materials. It has been developed for many years and the key research scope focuses on preparation and application of microPCMs with appropriate phase change temperatures and desirable thermal stability (5-7). PCMs achieve the storage and release of energy through the latent heat of phase change (3). At present, these materials have been widely used in textiles, building materials and some other fields $(3,8)$. Microspheres have attracted more attention in recent years. They have potential application in energy storage, thermal insulation, drug delivery, and some other fields (9-13). Microspheres can be fabricated by natural and synthetic polymers (13-16). There are many chemical and physicochemical methods available to fabricate microspheres (17-19).

Hexadecyl acrylate polymer (PHA) is a solid-liquid phase change material, which can absorb and release heat energy at $39^{\circ} \mathrm{C}$. However, it is not suitable for application since its unstable olefinic bond. It was used as a shell material in this paper, and further investigations were made to the effect of different crosslinking agents on the surface and phase transition properties of microspheres. In order to expand the application of PHA in a wider range of fields, we have prepared rapidrelease microspheres using phase change materials (20). In this research, microspheres with phase change properties were fabricated by polymerization of hexadecyl acrylate (HA) and different crosslinking agents, such as hexadecyl acrylate, allyl methacrylate, 1,4-butylene glycol diacrylate, trimethylolpropyltriacrylate, divinyl benzene, pentaerythritol tetraacrylate, which will help increase the strength and rigidity of the shell material. In addition, the $\beta$-tricalcium phosphate has good biocompatibility and can promote bio-bone repair, so it was encapsulated 
in microspheres to obtain one with a fast release effect. It will effectively promote bone conduction when these microspheres were implanted into a bone defect. This microsphere can be used for orthopedic implant or coating of instrument in the future.

\section{Experimental}

\subsection{Materials}

Hexadecyl acrylate (HA, purity, 98 wt\%, China Zhejiang Kangde Chemical Inc.) was used as the monomer in the composition of the shell. Allyl methacrylate (AMA, purity, $99.7 \mathrm{wt} \%$ ) was purchased from China Tianjin Jintongletai Chemical Product Corporation). 1,4-Butylene glycol diacrylate (BDDA; purity, $99.0 \mathrm{wt} \%$ ) and trimethylolpropyltriacrylate (TMPTA, purity, $99.0 \mathrm{wt} \%$ ) were products of China Liyang Blazer Chemical Technology Service Center. Divinyl benzene (DVB, purity, $55.0 \mathrm{wt} \%$ ) was purchased from China Tianjin Jintongletai Chemical Product Corporation. Pentaerythritol tetraacrylate (PETRA, purity, $99.0 \mathrm{wt} \%$ ) was also a product of China Liyang Blazer Chemical Technology Service Center, which was used as the crosslinking agents in the composition of the microspheres. 2,2'-azobisisobutyronitrile (AIBN, 98.0 wt\%, China Tianjin Zhiyuan Chemical Reagent Corporation) was employed as the initiator during the reaction. Sodium salt emulsion of styrene-maleic anhydride copolymer (Na-SMA, 19 wt $\%$ aqueous solutions, China Shanghai Leather Chemical Works) was used as surfactant. $\beta$-tricalcium phosphate was purchased from Aladdin Reagent (China Shanghai) Co., Ltd. (purity, 99.8 wt\%).

\subsection{Fabrication of microspheres}

The dispersed oil phase consists of $0.3 \mathrm{~g}$ 2,2'-azobisisobutyronitrile (AIBN), $0.02 \mathrm{~mol}$ hexadecyl acrylate (HA) and 0.01 mol crosslinking agents (Figure 1). The aqueous phase was fabricated by dissolving $100 \mathrm{~mL} \mathrm{H} 2 \mathrm{O}$ and $7 \mathrm{~g}$ sodium salt emulsion of styrenemaleic anhydride copolymer (Na-SMA). The oil phase was added into the aqueous phase under a stirring rate of $500 \mathrm{rpm}$. After being emulsified by a homogenizer at $1500 \mathrm{rpm}$ for $15 \mathrm{~min}$, the reaction was carried out for $6 \mathrm{~h}$ under a stirring rate of $550 \mathrm{rpm}$ at $85^{\circ} \mathrm{C}$. Finally, the product was washed with ethanol at $50^{\circ} \mathrm{C}$ and was vacuum-dried at $45^{\circ} \mathrm{C}$ for $24 \mathrm{~h}$.

\subsection{Characterization}

Fourier transform infrared spectroscopy (FTIR) spectra was recorded on a Bruker Vector 22 spectrometer (Germany) at natural temperature. The micrographs of the microsphere were observed by scanning electron microscope (SEM, Hitachi S4800, Japan), samples were coated with a layer of gold. Thermal Gravimetric Analysis (TGA) plots were obtained by thermogravimetry (NETZSCH STA 409 PC/ PGTG-DTA, Germany), with a heating rate of $10^{\circ} \mathrm{C} / \mathrm{min}$ in a nitrogen atmosphere, in the range of $20^{\circ} \mathrm{C}-800^{\circ} \mathrm{C}$. The thermal behavior of the microsphere was obtained using a differential scanning calorimeter (DSC, NETZSCH DSC $200 \mathrm{~F} 3$, Germany) in the range of from $-20^{\circ} \mathrm{C}$ to $80^{\circ} \mathrm{C}$ at a heating or cooling rate of $10^{\circ} \mathrm{C} / \mathrm{min}$ in a nitrogen atmosphere. Particle size distribution of the microspheres was obtained by a laser particle size analyzer (Horiba LA-300, Japan). X-ray photoelectron spectroscopy (XPS, K-alpha, Thermofisher, United States) was used for element analysis.

\section{Results and discussion}

\subsection{Chemical characterization}

In Figure 2, compared with PHA, the bands at $2926 \mathrm{~cm}^{-1}$ and $2856 \mathrm{~cm}^{-1}$ are assigned to stretching vibration of $-\mathrm{CH}_{3}$ and $-\mathrm{CH}_{2}$, respectively. The bands corresponding to $\mathrm{C}-\mathrm{H}$ stretching vibration of polymer shells are overlapped with hexadecyl acrylate structural unit in each microsphere spectrum. The band at $1468 \mathrm{~cm}^{-1}$ is assigned to the deformation vibration of $-\mathrm{CH}_{2}$. The bands at around $1468 \mathrm{~cm}^{-1}$ and $1410 \mathrm{~cm}^{-1}$, which can be observed in both hexadecyl acrylate structural unit spectrum and each microspheres spectrum, and $717 \mathrm{~cm}^{-1}$ are attributed to a long-chain horizontal rocking vibration absorption peak containing more than four $-\mathrm{CH}_{2-}$ groups in the molecule, these are the characteristic bands for hexadecyl acrylate structural unit. The bands at $1735 \mathrm{~cm}^{-1}$ and $1162 \mathrm{~cm}^{-1}$ are assigned to $\mathrm{C}=\mathrm{O}$ stretching vibration and $\mathrm{C}-\mathrm{O}$ stretching of the ester group in the polymer shell, due to the presence of the crosslinked structure, the peak at that point appears offset, this may be due to the induced effect causing a change in the distribution of electron clouds in the molecule, causing a change in the bond force constant, which shifts the frequency of the group. The band at $1497 \mathrm{~cm}^{-1}$ observed in the spectra of HA-DVB polymer can be assigned to the benzene ring bending vibration. 
<smiles>C=CCOC(=O)C(=C)C</smiles>

A

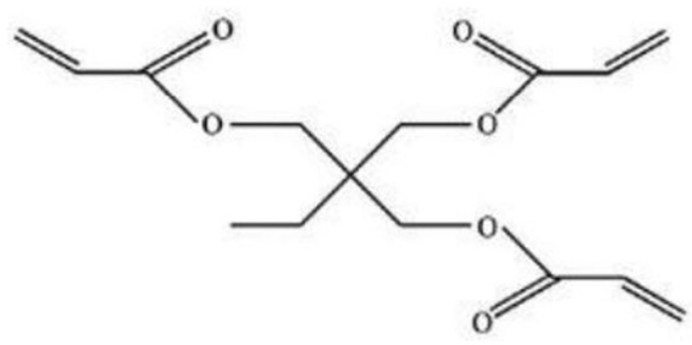

D

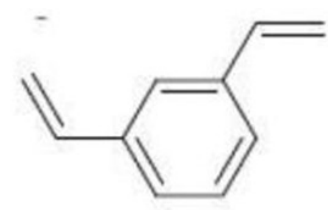<smiles>C=CC(=O)OCCCCOC(=O)C=C</smiles>

B

$\mathrm{C}$

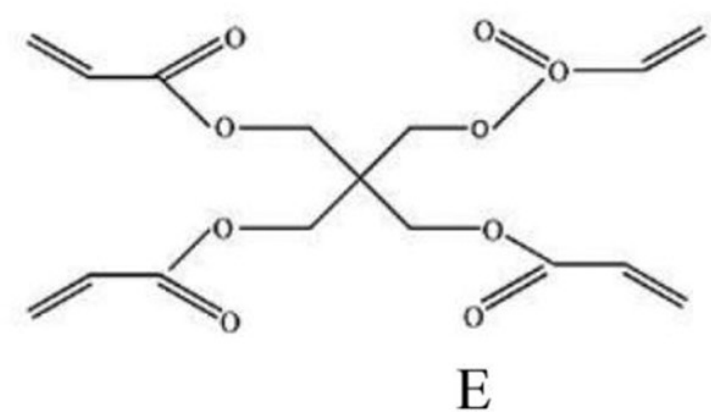

Figure 1: The chemical structure of cross-linking agents: (a) allyl methacrylate (AMA), (b) divinyl benzene (DVB), (c) 1,4-butylene glycol diacrylate (BDDA), (d) trimethylolpropyltriacrylate (TMPTA), (e) pentaerythritol tetraacrylate (PETRA)

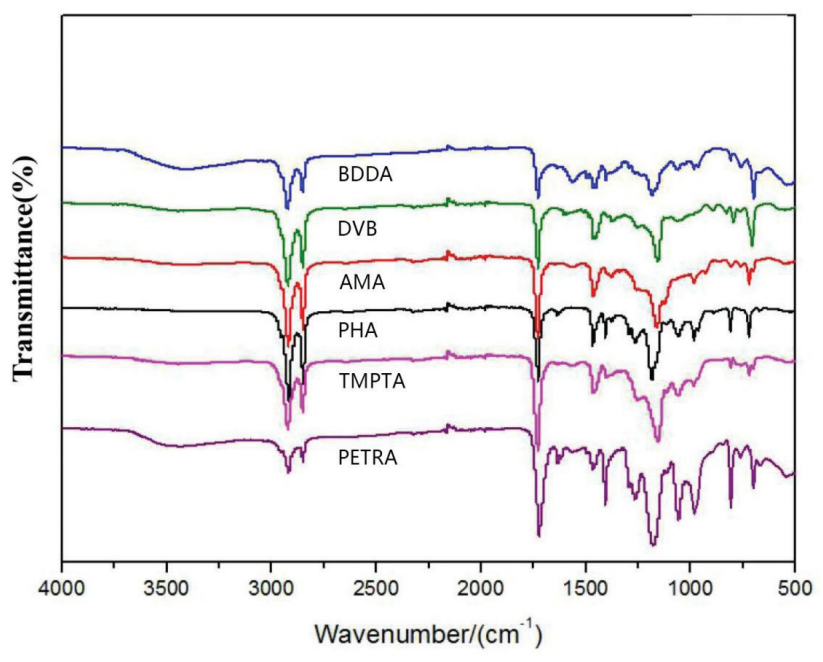

Figure 2: FTIR spectra of the microspheres with various cross-linking agents.

The FTIR test results showed that the monomers had effective polymerization reaction and the target products were obtained.

The particle size distributions of the microspheres are shown in Figure 3. The particle size ranged mainly from $25 \mu \mathrm{m}$ to $100 \mu \mathrm{m}$ for HA-AMA, HA-TMPTA and HA-PETRA microspheres, and from $3 \mu \mathrm{m}$ to $10 \mu \mathrm{m}$ for the HA-BDDA and HA-DVB microspheres. The particle size distributions of the five microspheres are different. This may be caused by the difference of the surface tension between the organic phase and the aqueous phase.

\subsection{Morphology of microspheres}

SEM micrographs of the surface of various micrographs are shown in Figure 4. The capsules show a regular spherical shape except the shell that obtained only by PHA. It is observed that the surface of the micrographs is spherical and smooth, without any defects, this indicates that the addition of cross-linking agent has no obvious effect on the surface morphology of microspheres and this will be beneficial to the application of microspheres in the field of phase change material, washing materials and drugs.

\subsection{Thermal properties and thermal stability}

Thermal properties of microspheres fabricated with different cross-linking agents are shown in Figure 5 and summarized in Table 1. As shown in the curve, the endothermic peaks of microspheres fabricated with AMA, BDDA, TMPTA and PETRA as crosslinking agents decreased slightly, the endothermic peaks of microspheres fabricated with DVB as crosslinking agents significantly improved by $2.3^{\circ} \mathrm{C}$. This phenomenon shows the phase transition temperature of the microspheres decreased with the increase of functional groups of the cross-linking agents. This is because cross-linking agents with complex structures destroy the regularity of the macromolecular chains and this is not conducive to crystallization. In addition, microspheres fabricated using DVB has the 


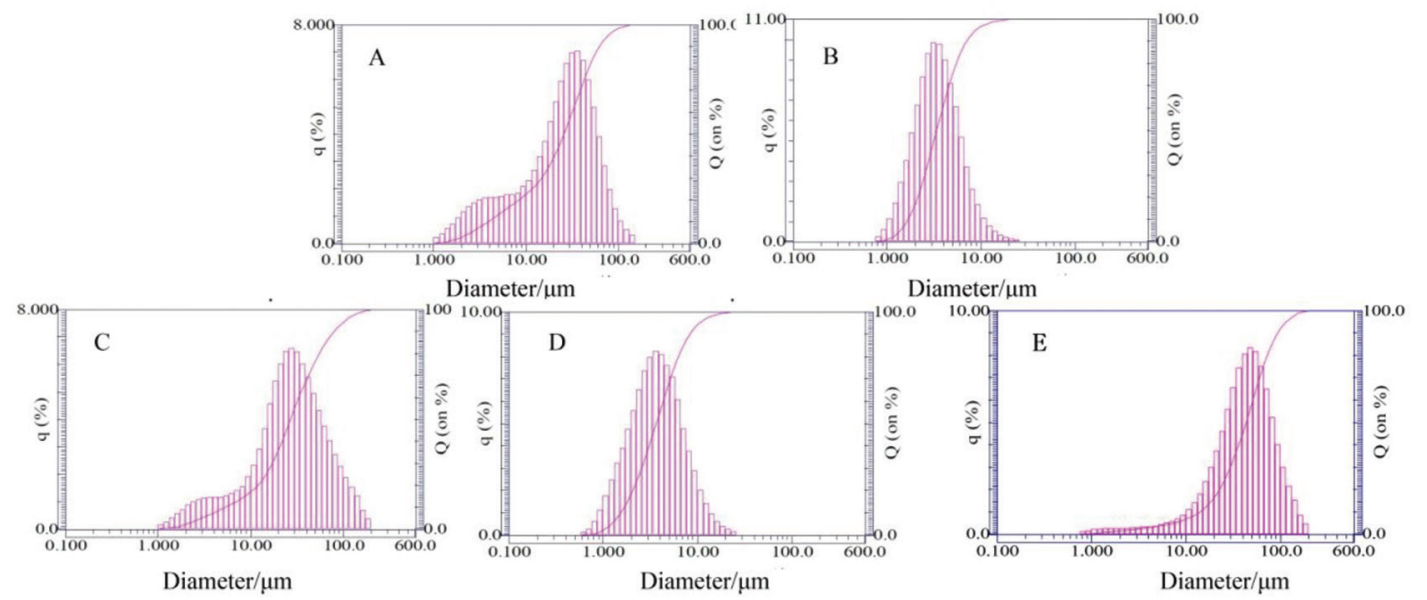

Figure 3: Particle size distribution of the microspheres: (a) HA-AMA, (b) HA-BDDA, (c) HA-PETRA, (d) HA-DVB, (e) HA-TMPTA.

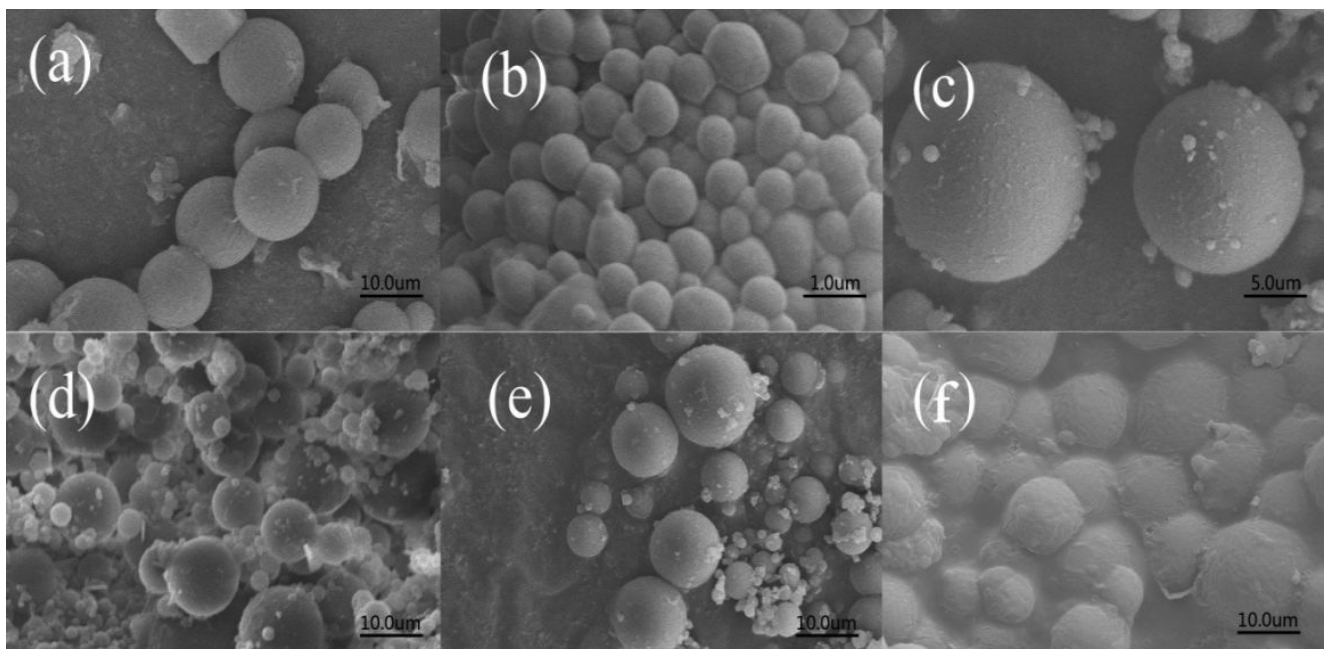

Figure 4: SEM micrographs of (a) HA-AMA, (b) HA-BDDA, (c) HA-PETRA, (d) HA-TMPTA, (e) HA-DVB, (f) PHA.

highest latent heats of melting. The results show that the microspheres fabricated by HA and DVB have better structural regularity and application potential. HA-TMPTA microspheres did not show significant endothermic peaks and exothermic peaks, it indicates that the presence of TMPTA affected the crystallinity of the material and leaded to a decrease in the crystallinity of the microspheres.

Thermal stability is one of significant factors in evaluating microspheres for their applications. Figure 6 shows the TGA plots of PHA, HA-AMA, HA-BDDA, HA-TMPTA, HA-PETRA and HA-DVB shells. The extracted value at the temperature for occurrence of mass loss of $5 \mathrm{wt} \%$ (Td5\%), and the temperature of HA-AMA is approximately $40^{\circ} \mathrm{C}$ above that of PHA. It is believed that the cross-linked copolymer shell exhibits better thermal stability than that of PHA. The thermal decomposition temperature of PHA shell is approximately equivalent to that of the cross-linked copolymer shells. There is no obvious difference between the shells.

\subsection{Application}

To verify the temperature controlled release properties of HA-AMA, HA-BDDA, HA-PETRA, HA-TMPTA and HA-DVB, microspheres were prepared using $\beta$-tricalcium phosphate as core materials. The release principle of temperature controlled release microspheres is simple and the microspheres can be effectively controlled by temperature. This kind of microsphere can be widely used in food, medical, construction, textile, daily chemical and other fields. The experiment in Figure 7 validates the actual release effect of temperature controlled release microspheres. The microspheres are placed in 


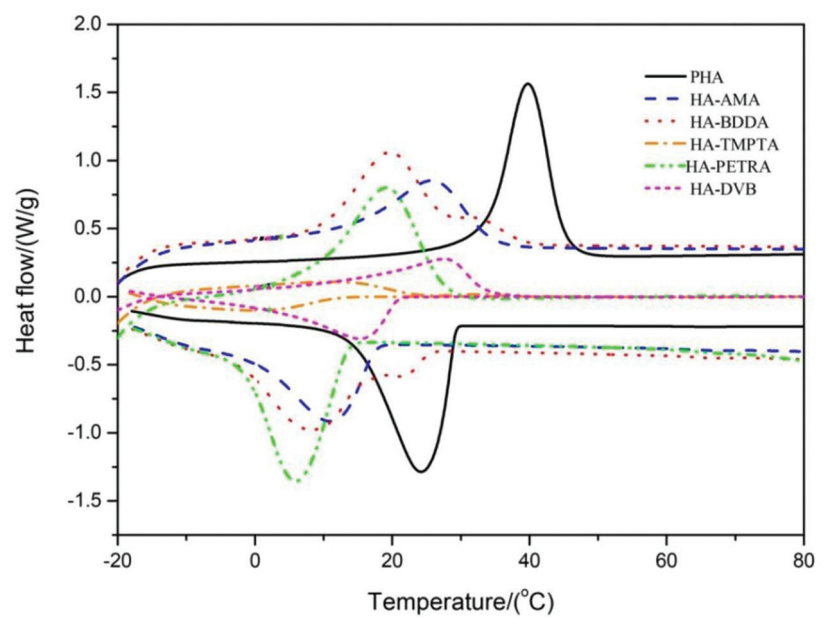

Figure 5: DSC curve of microspheres with various cross-linking agents.

Table 1: Thermal properties of microspheres with various crosslinking agents.

\begin{tabular}{lrccccc}
\hline Material & \multicolumn{1}{c}{$\mathrm{T}_{\mathrm{om}} /{ }^{\circ} \mathrm{C}$} & $\mathrm{T}_{\mathrm{pm}} /{ }^{\circ} \mathrm{C}$ & $\Delta \mathrm{Hm} / \mathrm{Jg}^{-1}$ & $\mathrm{~T}_{\mathrm{oc}} /{ }^{\circ} \mathrm{C}$ & $\mathrm{T}_{\mathrm{pc}} /{ }^{\circ} \mathrm{C}$ & $\Delta \mathrm{Hc} / \mathrm{Jg}^{-1}$ \\
\hline PHA & 33.9 & 39.8 & 116.4 & 29.3 & 24.2 & 115.9 \\
HA-AMA & 14.7 & 25.8 & 42.03 & 17.5 & 10.9 & 41.83 \\
HA-BDDA & 10.0 & 19.7 & 58.56 & 14.9 & 8.2 & 58.54 \\
HA-TMPTA & 2.7 & 16.4 & 24.60 & 15.4 & 6.1 & 25.64 \\
HA-PETRA & 9.4 & 19.1 & 63.64 & 13.4 & 5.9 & 63.28 \\
HA-DVB & 18.3 & 31.6 & 70.68 & 28.4 & 15 & 69.03 \\
\hline
\end{tabular}

Note: $\mathrm{T}_{\text {om }}$ - on set temperature on DSC heating curve; $\mathrm{T}_{\mathrm{pm}}$ - peak temperature on DSC heating curve;

$\triangle \mathrm{Hm}$ - enthalpy on DSC heating curve; $\mathrm{T}_{\text {oc }}$ - on set temperature on DSC cooling curve;

$\mathrm{T}_{\mathrm{pc}}$ - peak temperature on DSC cooling curve; $\Delta \mathrm{Hc}$ - enthalpy on DSC cooling curve.

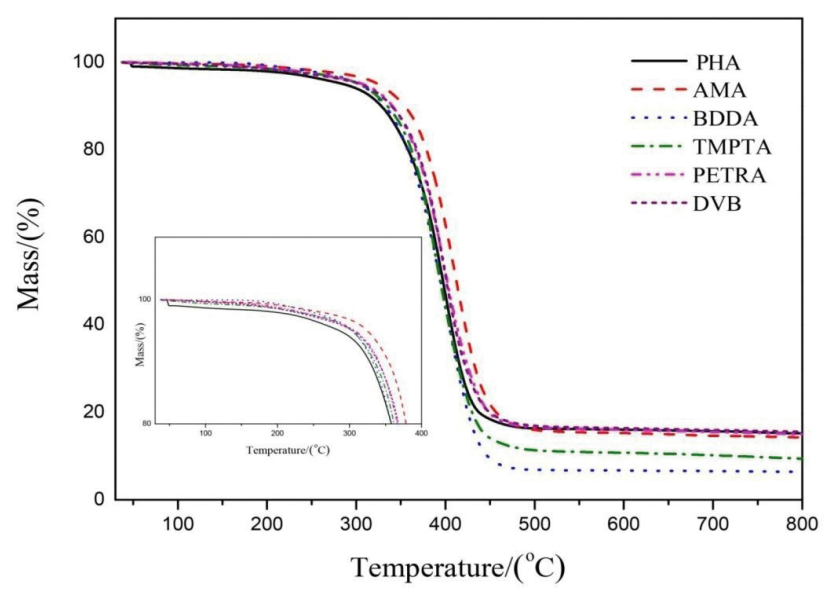

Figure 6: TG curve of the microspheres with various cross-linking agents. the water bath. When the temperature is lower than the phase transition temperature of the shell material, the microspheres are suspended in the water and after the temperature is increased to the phase transition temperature, the shell material undergoes a phase change and the microspheres gradually rupture and form the film. The core material sank at the bottom of the tube after the core material was released. The release process of various microcapsules is recorded as shown in Figure 7. Due to the different phase transition temperatures of the microcapsule shell materials, the melting and release rates of the microcapsules are different. The release times of HA-TMPTA, HA-PETRA, HA-BDDA, HA-AMA and HA-DVB were 86 s, 93 s, 98 s, 116 s and 142 s, respectively.

It can be seen from the SEM image in Figure 8 that the HA-PETRA microspheres were released after the particles were melted. And the ratio of $\mathrm{Ca} / \mathrm{P}$ of the particles analyzed by EDS is 1.67. In addition, the XPS elemental analysis of the HA-PETRA microcapsules with completed release is carried out as shown in Figure 9, and the $\mathrm{Ca} / \mathrm{P}$ ratio is 1.30, which is closed to the test result of EDS, so these results indicating that the released particles were $\beta$-tricalcium phosphate. The separation phenomenon of the core and the shell indicates that HA-AMA, HA-BDDA, HA-PETRA, HA-TMPTA and HA-DVB can be used as shell materials to realize the preparation of microspheres and have obvious temperature controlled release effect. This verification is of great significance for carrying out the later research and application.

\section{Conclusions}

In this study, microspheres could be obtained by using AMA, BDDA, PETRA, DVB and TMPTA as the cross-linkers. All of the cross-linked copolymer shells exhibit better thermal stability than that of PHA. The latent heats of microspheres fabricated by various cross-linking agents are different. The cross-linking agents with complex structures destroy the regularity of the macromolecular chains and it will affect the crystallization of microspheres but it has no effect on their surface morphology. The latent heat of microspheres fabricated by DVB as cross-linker is the largest and the particle size of the microsphere is uniform. Since the crystal structure is destroyed by varying degrees, the various microspheres have different lower phase transition temperatures, so that all of the cross-linked copolymer shells can be made into temperature controlled release microspheres and the experiments show that all kinds of microcapsules have significant temperature-controlled release effect. 


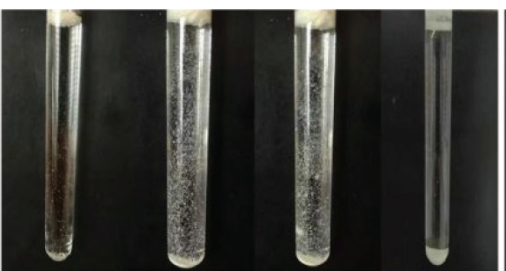

HA-AMA

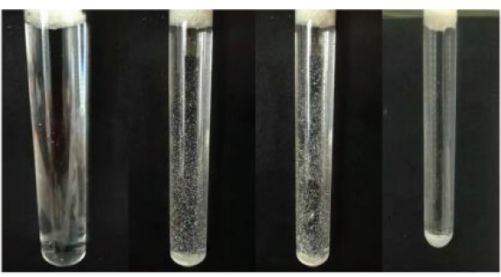

HA-DVB

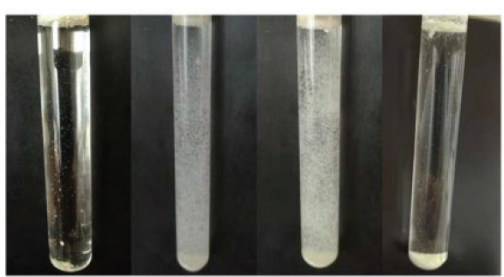

HA-BDDA

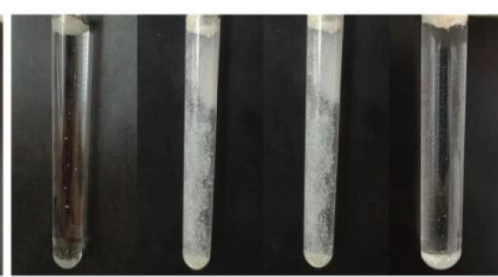

HA-TMPTA

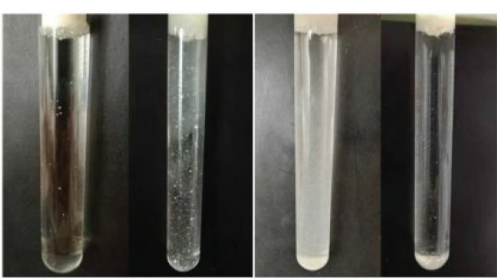

HA-PETRA

Figure 7: Release effect verification experiment of the temperature controlled release microspheres.

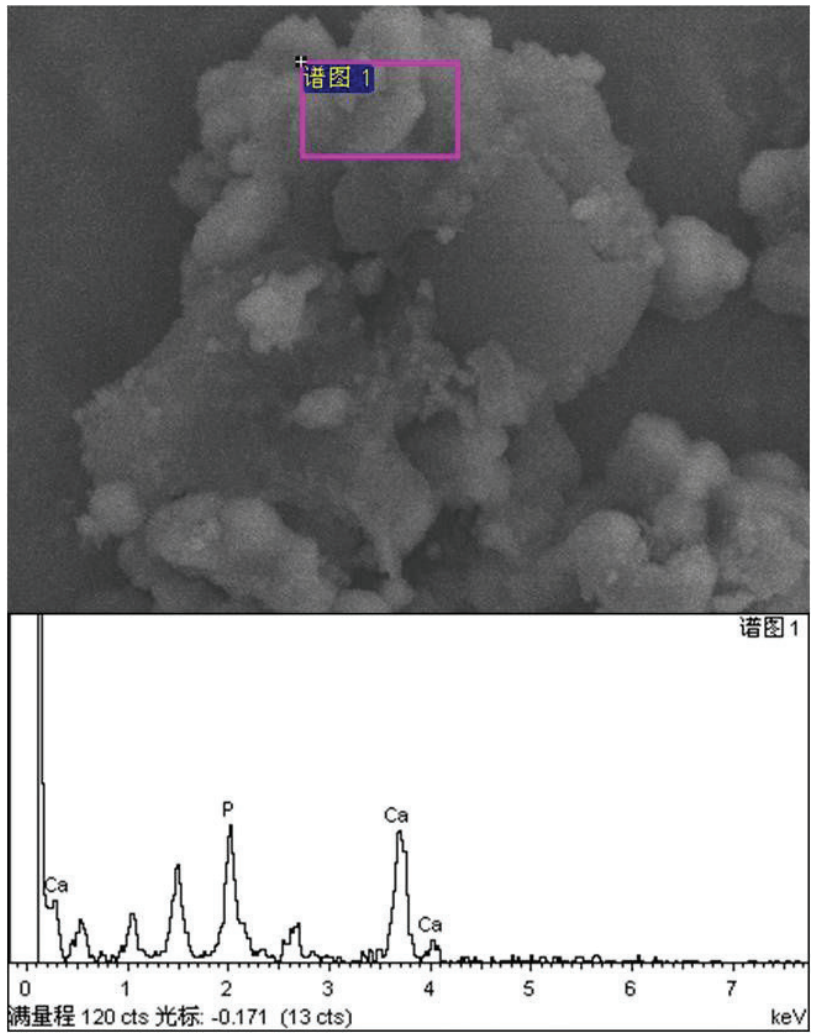

Figure 8: SEM picture and EDS analysis of HA-PETRA microspheres after melting.

Acknowledgment: The work was supported by the New Materials Research Key Program of Tianjin (No. 16ZXCLGX00090), the National Key Research and Development Program of China (No. 2016YFB0303000) and State Key Laboratory of Separation Membranes and Membrane Processes.

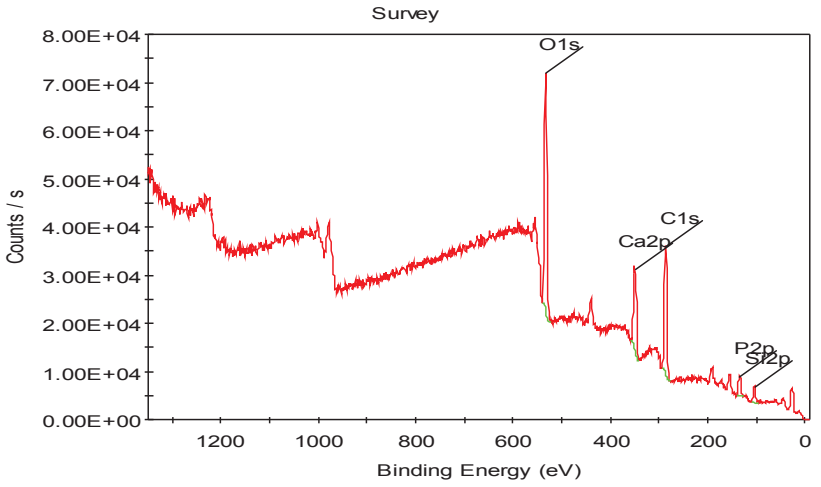

Figure 9: XPS analysis of HA-PETRA microcapsules after release of $\beta$-tricalcium phosphate.

\section{References}

1. Li M.G., Zhang Y., Effect of different amounts of surfactant on characteristics of nano-encapsulated phase-change materials. Polym. Bull., 2011, 67, 541-552.

2. Zhang L., Jin Y., Liu H., Du. Y., Structure and control release of chitosan/carboxymethyl cellulose microspheres. J. Appl. Polym. Sci., 2001, 82, 584-592.

3. Sharma A., Tyagi V.V., Chen C.R., Buddhi D., Review on thermal energy storage with phase change materials and applications. Renew. Sust. Energ. Rev., 2009, 13(2), 318-345.

4. Ahmed A., Sanada K., Fanni M., El-Moneim A., A practical methodology for modeling and verification of self-healing microspheres-based composites elasticity. Compos. Struct., 2018, 184, 1092-1098.

5. Palanikkumaran M., Gupta K.K., Agrawal A.K., Jassal M., Highly stable hexamethylolmelamine microcapsules containing n-octadecane prepared by in situ encapsulation. J. Appl. Polym. Sci., 2009, 114, 2997-3002.

6. Yu F., Chen Z.-H., Zeng X.-R., Gao X.-N., Zhang Z.-G., Poly(methyl methacrylate) copolymer nanocapsules containing phase-change 
material (n-dodecanol) prepared via miniemulsion polymerization. J. Appl. Polym. Sci., 2015, 132, 42334

7. Szczotok A.M., Carmona M., Kjøniksen A.-L., Rodriguez J.F., The role of radical polymerization in the production of thermoregulating microcapsules or polymers from saturated and unsaturated fatty acids. J. Appl. Polym. Sci., 2018, 135, 45970.

8. Ahmed A., Sanada K., Fanni M., El-Moneim A.A., A practical methodology for modeling and verification of self-healing microcapsules-based composites elasticity. Compos. Struct., 2018, 184, 1092-1098.

9. Liu J., Detrembleur C., De Pauw-Gillet M.C., Mornet S., Jérôme C., Duguet E., Gold Nanorods Coated with Mesoporous $\beta$-tricalcium phosphate Shell as Drug Delivery System for Remote Near Infrared Light-Activated Release and Potential Phototherapy. SMALL, 2015, 11, 2323-2332.

10. Nejman A., Cieślak M., The impact of the heating/cooling rate on the thermoregulating properties of textile materials modified with PCM microspheres. Appl. Therm. Eng., 2017, 127, 212-223.

11. Delgado M., Lázaro A., Peñalosa C., Zalba B., Experimental analysis of the influence of microsphere mass fraction on the thermal and rheological behavior of a PCM slurry. Appl. Therm. Eng., 2014, 63, 11-22.

12. Konuklu Y., Ostry M., Paksoy H., Charvat P., Review on using microencapsulated phase change materials (PCM) in building applications. Energ. Buildings, 2015, 106, 134-155.

13. Aguayo M., Das S., Maroli A., Kabay N., Mertens J.C.E., Rajan S.D., et al., The influence of microencapsulated phase change material (PCM) characteristics on the microstructure and strength of cementitious composites: Experiments and finite element simulations. Cement Concrete Comp., 2016, 73, 29-41.

14. Ahangaran F., Hayaty M., Navarchian A., Picchioni F., Micromechanical assessment of PMMA microspheres containing epoxy and mercaptan as self-healing agents. Polym. Test., 2017, 64, 330-336.

15. Tang X F., Li W., Zhang X.X., New approach to fabricate microspheres with comb-like copolymer shell by phase separation method. Adv. Mater. Res., 2014, 860-863, 577-581.

16. Sarı A., Biçer A., Karaipekli A., Synthesis, characterization, thermal properties of a series of stearic acid esters as novel solid-liquid phase change materials. Mater. Lett., 2009, 63, 1213-1216.

17. MaY., ChuX., Tang G., Yao Y., Adjusting phase change temperature of microspheres by regulating their core compositions. Mater. Lett., 2012, 82, 39-41.

18. Ma Y., Chu X., Tang G., Yao Y., Synthesis and thermal properties of acrylate-based polymer shell microspheres with binary core as phase change materials. Mater. Lett., 2013, 91, 133-135.

19. Qiu X., Li W., Song G.L., Chu X.G., Tang G.Y., Fabrication and characterization of microencapsulated n-octadecane with different crosslinked methyl methacrylate-based polymer shells. Sol. Energ. Mater. Sol. C., 2012, 98, 283-293.

20. Ahangaran F., Hayaty M., Navarchian A.H., Picchioni F., Micromechanical assessment of PMMA microcapsules containing epoxy and mercaptan as self-healing agents. Polym. Test., 2017, 64, 330-336. 